\title{
Degree of MWCNT suspension dispersity and its influence on rheology of cement pastes
}

\author{
Gintautas Skripkiunas ${ }^{1}$, Ekaterina Karpova ${ }^{2}$, Joana Bendoraitiene ${ }^{3}$, \\ Irmantas Barauskas ${ }^{4}$, Rostislav Drochytka ${ }^{5}$ \\ ${ }^{1,2}$ Department of Building Materials and Fire Safety, Vilnius Gediminas Technical University, Vilnius, Lithuania \\ ${ }^{3}$ Department of Polymer Chemistry and Technology, Kaunas University of Technology, Kaunas, Lithuania \\ ${ }^{4}$ Department of Silicate Technology, Kaunas University of Technology, Kaunas, Lithuania \\ ${ }^{5}$ Department of Technology of Building Materials and Components, Brno University of Technology, \\ Brno, Czech Republic
}

\section{E-mail: 2ekaterina.karpova@vgtu.lt (corresponding author)}

\begin{abstract}
Modification of cement pastes by MWCNT (multi-walled carbon nanotubes) suspension enables to manage by their rheological properties due to the nanometer size of MWCNT. Dispersity degree of MWCNT suspension may be determined by the number of factors such as type and intensity of homogenization equipment, duration of homogenization and some other. The aim of present research consists in the establishment of optimal methodology of MWCNT suspension homogenization and investigation of dependence between dispersity degree of MWCNT suspension and rheological properties of cement pastes with that suspension. Two types of homogenization methods of MWCNT were tested such as ultrasonication and homogenization based on rotor-stator principle. The ultrasonication treatment about 6 min was established as optimal homogenization technique in the course of the current research. The yield stress and plastic viscosity of cement paste modified by MWCNT suspension increased in comparison with cement paste modified by polycarboxylate ether plasticizer (PCE) in 5 and $30 \mathrm{~min}$ after cement pastes mixing. Time of ultrasonication of MWCNT suspension more than $6 \mathrm{~min}$ did not have the influence on rheological behaviour of cement pastes.Addition of MWCNT decreased the volume coefficient of water bleeding of cement paste with PCE by $18 \%$.
\end{abstract}

Keywords: multi-walled-carbon nanotubes, cement paste, rheology, dispersity degree, homogenization.

\section{Introduction}

Fundamental rheological parameters of cement systems, such as yield stress, thixotropy and viscosity, depends on water content, cement composition, aggregates particle size distribution, type of applied additives and admixtures, time after mixing of cement systems, type of mixing during the test and some other properties (Yahia, Mantellato, \& Flatt, 2016; Jiao et al., 2017; Secrieru et al., 2018).

The particle size of constituents in cement systems and interactions between them are one the key factors which determine the rheological behavior and technological properties of mixtures. Hydrodynamic, colloidal, inertial, gravitational forces and Brownian motion underlie the interactions between particles in cement systems (Roussel, Lemâ̂tre, Flatt, \& Coussot, 2010; Ma, Qian, \& Kawashima, 2018a).

The size, shape, distance between aggregates in concrete and mortars have an influence on the rheological properties of cement systems. The influence of sand content on rheological properties of concrete mixtures was analyzed in the (Dauksys \& Skripkiunas, 2018). The increase of sand content from 50 to $65 \%$ increased the yield stress approximately 1.5 times, decreased viscosity and dilatancy index and led to higher workability of concrete mixture.

Widespread application of mineral additives and chemical admixtures for generation of high workability and self-compacting concrete in construction practice requires the understanding of their action mechanism in cement systems (Bentz, Ferraris, Galler, Hansen, \& Guynn, 2012; Štefančič, Mladenovič, Bellotto, Jereb, \& Završnik, 2017; Vance, Kumar, Sant, \& Neithalath, 2013; Zhang, Luo, Kong, Wang, \& Gao, 2018; Qian et al., 2018; Smirnova, 2018a, 2018b). Development of self-compacting concrete (SCC) requires not only application of effective polymer plasticizers to reach high flowability, but also chemical admixtures to provide mixture stability (Isik \& Ozkul, 2014). Nanoadditives with nanometer dimensions of particles, high aspect ratio and unique physical, chemical, electrical

(C) 2019 Authors. Published by VGTU Press. This is an open-access article distributed under the terms of the Creative Commons Attribution (http://creativecommons.org/licenses/by/4.0/) License, which permits unrestricted use, distribution, and reproduction in any medium, provided the original author and source are credited. 
properties may be used as a perspective tool to improve the stability of concrete mixture. With an increase of content of graphite nanoplatelet and carbon nanofiber from 0.05 to $0.30 \%$ by weight of binder, W. Meng et al. obtained the increase of plastic viscosity (Meng \& Khayat, 2018).

Nano-silica can be used as thickening agent of cement paste (Lavergne, Belhadi, Carriat, \& Ben Fraj, 2019). E. Garcia-Taengua et al. observed the increase of viscosity of fresh mortar modified by $2 \%$ of nano-silica (García-Taengua, Sonebi, Hossain, Lachemi, \& Khatib, 2015).

Application of cellulose filaments (CF) with diameter of 30-400 nm, length of 100-2000 nm and aspect ratio of $100-1000 \mathrm{~nm}$ for modification of cement pastes and SCC demonstrated the significant viscosity modifying effect in dosage of CF from 0.05 to $0.30 \%$ by weight of binder (Hisseine, Basic, Omran, \& Tagnit-Hamou, 2018).

Carbon nanotubes (CNT) increase all rheological parameters in cement mortar. However, their application in blended systems may have the opposite effect on rheology (Ma, Qian, \& Kawashima, 2018b). Frequently, carbon nanotubes are used in combination with dispersants applied for their better distribution. The researchers (Nadiv et al., 2016; Mendoza Reales et al., 2018) highlighted the importance of interactions between dispersing agent and CNT.

The main barrier in wide application of nanomaterials in construction practice caused by their distribution inside volume of water suspension and cement systems. The effectiveness of homogenization is directly correlated with dispersion degree of generated suspension and has the further influence on rheological properties of cement systems modified by nanoadditives. Homogenization of nanomaterials can be provided by different methods, such as bead milling process (Yoshio et al., 2011), hydrodynamic, acoustic cavitation (Chai, Yusup, Chok, Arpin, \& Irawan, 2016; Kiu, Yusup, Soon, Arpin, \& Samion, 2016) and ultrasonication technique, which is more frequently used nowadays (Zou et al., 2015; Konsta-Gdoutos, Metaxa, \& Shah, 2010). The listed methods allow to avoid agglomeration process in water suspension of nanoparticles and make it possible to use them for modification of cement systems in fresh and hardened state.

The aim of current research is to compare ultrasonication method and method based on rotor-stator principle for homogenization of MWCNT suspension with establishment of optimal technique and further investigation of dependence between dispersity degree of MWCNT suspension and rheological properties of cement pastes modified with that suspension.

\section{Materials and Methods}

\section{Materials}

The Portland cement without mineral additives CEM I 42.5 R conforming to EN 197-1 with water consumption $26.6 \%$ and fineness by Blain of $3552 \mathrm{~cm}^{2} / \mathrm{g}$ was used as a binder.

The curve of cement particle size distribution, determined by CILAS 1090, is presented in Figure 1a. The main content of particles of cement is placed in the range from 1 to $80 \mu \mathrm{m}$ in size with average value of cement particles size about $14.28 \mu \mathrm{m}$.

a)

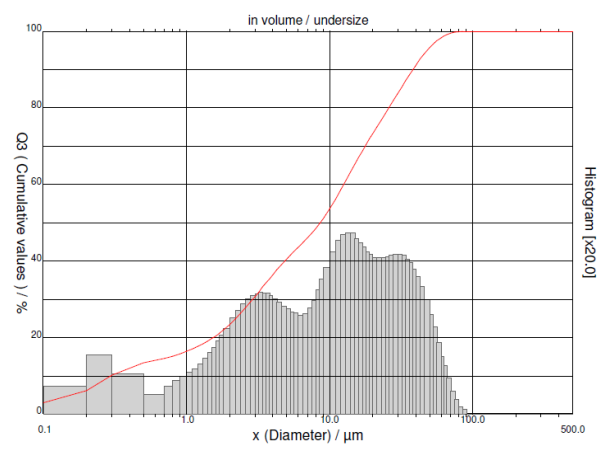

b)

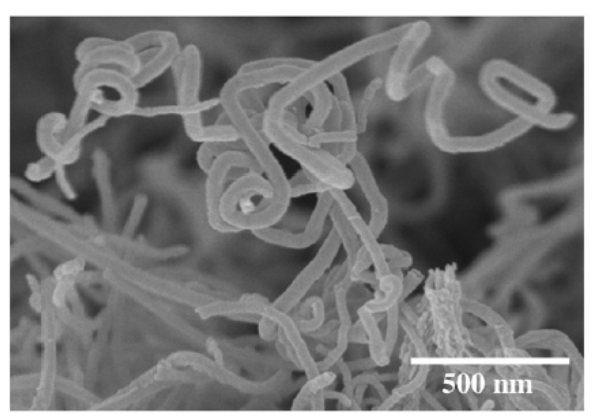

Figure 1. The particle size distribution of Portland cement without mineral additives(a); SEM image of MWCNT (b)(source: (Zou et al., 2015))

The MWCNT suspensionwas prepared from the masterbatch pellets "Graphistrength CW 2-45"produced by the company "Arkema" (France). The masterbatch contains 45 wt. \% of MWCNT and 55 wt. \% of carboxymethylcellulose (CMC). The MWCNT was characterized by filament length of $100-10000 \mathrm{~nm}$ and diameter of 15-20 nm. SEM image of MWCNT is presented in Figure 1b. Distilled water was used as dispersion medium for MWCNT. The polycarboxylate ether of MPEG type (PCE) with specific gravity $1.100 \mathrm{~g} / \mathrm{cm}^{3}, \mathrm{pH}$ up to 5 at $20^{\circ} \mathrm{C}$ and dry content equal to $50.5 \%$ was applied in the research as dispersant to distribute the nanotubes in the volume of suspension. 
Table 1. Composition of MWCNT suspensions

\begin{tabular}{|c|c|c|c|c|}
\hline Specimen & $\begin{array}{c}\text { MWCNT in volume } \\
\text { of suspension, \% }\end{array}$ & $\begin{array}{c}\text { PCE in volume of } \\
\text { suspension, \% }\end{array}$ & $\begin{array}{c}\text { Ratio between } \\
\text { MWCNT and PCE }\end{array}$ & $\begin{array}{c}\text { Time of ultrasonication /rotor-stator } \\
\text { homogenization, min }\end{array}$ \\
\hline \multicolumn{7}{|c|}{ Ultrasonication } \\
\hline S0 & 0.28 & 1.12 & $1: 4$ & 0 \\
\hline S3 & 0.28 & 1.12 & $1: 4$ & 6 \\
\hline S6 & 0.28 & 1.12 & $1: 4$ & 12 \\
\hline S9 & 0.28 & 1.12 & $1: 4$ & 6 \\
\hline S12 & 0.28 & 1.12 & $1: 4$ & 6 \\
\hline \multicolumn{7}{|c|}{} \\
\hline ST6 & 0.28 & Rotor-stator homogenization & 30 \\
\hline ST30 & 0.28 & 1.12 & $1: 4$ & $1: 4$ \\
\hline
\end{tabular}

The compositions of generated MWCNT suspensions are presented in Table 1. The ratio of 1 to 4 between MWCNT and PCE was set based on results of literaturereview.

\section{Methods}

The MWCNT suspension was prepared in following way. The masterbatch pellets "Graphistrength CW 2-45" were immersed in hot water with temperature about $75^{\circ} \mathrm{C}$ and stirred by standard mixer agitation about $1000 \mathrm{rpm}$ during 5 min.

After that the MWCNT suspension was subjected to ultrasonication for a duration of 3, 6, 9, 12 min. The ultrasonication was performed by Bandelin Sonopuls HD 3400 ultrasonic homogenizer ( $400 \mathrm{~W}, 20 \mathrm{kHz})$ with probe VS $200 \mathrm{~T}(\varnothing 25 \mathrm{~mm}$, amplitude $-82 \mu \mathrm{m})$. The time of ultrasonication was taken according to the data presented in (Liebscher et al., 2017), where the same ultrasonication equipment was used.

The ultrasonication technique was compared with method based on rotor-stator principle, which was recommended by nanotubes producer "Arkema". Rotor-stator homogenization was performed by Ultra-Turrax Dispersers T25 digital with frequency $50 / 60 \mathrm{~Hz}$ at speed $5000 \mathrm{rpm}$ for a duration 6 and $30 \mathrm{~min}$. The duration of treatment was selected based on the technical data sheet.

The utrasonication treatment is based on generation of ultrasonication waves, which promote to the dispergation of nanoparticles in the volume of water suspension. On the other hand, the rotor-stator equipment for homogenization consists of a rotor within a stationary stator. The high speed and minimal gap between the rotor and stator provide the generation of strong shear forces which lead to homogenization of particles.

The prepared MWCNT suspension was characterized by $\mathrm{pH}$, electrical conductivity tests, measurements of surface tension, particle size analysis and measurements of zeta potential. $\mathrm{pH}$ test was performed by $\mathrm{pH}$-meter Mettler-Toledo EL20 with limits of error $\pm 0.01 \mathrm{pH}$. The electrode with gel electrolyte was used.Electrical conductivity was measured by Mettler-Toledo EL30. The measurement range of it is 0.1 to $199.1 \mathrm{mS} / \mathrm{cm}$ with limits of error $\pm 0.5 \%$. The electrode for medium to high conductivities $(10 \mu \mathrm{S} / \mathrm{cm} \ldots 500 \mathrm{mS} / \mathrm{cm})$ was applied.

Surface tension of MWCNT suspension was measured based on stalagmometric method by stalagmometerTraubewith measurement accuracy $1 \mathrm{mN} / \mathrm{m}$. The stalagmometric method consists in determination ofdrops number which are formed in the course the flowing of liquid fromcapillary tube of known volume. The surface tension of studied liquid is calculated based on formula (1):

$$
\sigma_{x}=\sigma \cdot \frac{N}{N_{x}} \cdot \frac{d_{x}}{d}
$$

where: $\sigma$ - surface tension, $\mathrm{mN} / \mathrm{m} ; N$ - number of drops; $d$ - density, $\mathrm{g} / \mathrm{cm}^{3}$ ofwater; $\sigma_{x}, N_{x}, d_{x}$ - the same for studied liquid.

Particle size analysis and zeta potential measurements of MWCNT suspension were performed by particle size and zeta potential analyser Delsa Nano C, Beckman Coulter.Particle size analysiswas based on the principle of photon correlation spectroscopy with resolution of measurements placed from $0.6 \mathrm{~nm}$ to $7 \mu \mathrm{m}$ and measurement accuracy of $0.05 \mathrm{~nm}$.Zeta potential measurementswas based on the electrophoretic light scattering principle with range of measurement from $-150 \mathrm{mV}$ to $+150 \mathrm{mV}$ and measurement accuracy of $0.005 \mathrm{mV}$. Polidispersity index (PI) was calculated as ratio between the square of standard deviation and the square of mean diameter.

The cement pastes with composition presented in Table 2 were prepared for rheological test. 
Table 2. Content of materials for $1 \mathrm{~m}^{3}$ in tested cement pastes

\begin{tabular}{|c|c|c|c|c|c|}
\hline Specimen & Cement, $\mathrm{kg}$ & Water, $\mathrm{kg}$ & W/C ratio & MWCNT, \% bwoc* & PCE, \% bwoc* \\
\hline REF & 935 & 280 & 0.30 & 0 & 0 \\
\hline PCE & 935 & 280 & 0.30 & 0 & 0.3 \\
\hline S3 & 935 & 280 & 0.30 & 0.08 & 0.3 \\
\hline S6 & 935 & 280 & 0.30 & 0.08 & 0.3 \\
\hline S9 & 935 & 280 & 0.30 & 0.08 & 0.3 \\
\hline S12 & 935 & 280 & 0.30 & 0.08 & 0.3 \\
\hline
\end{tabular}

*bwoc - by weight of cement.

The MWCNT suspensions with composition presented in Table 1 were used as mixing liquidandmixed together with cement for $180 \mathrm{~s}$ at high speed by standard mixeraccording to EN 196-1.

Rheological properties of cement pastes were tested in different time after pastes mixing: 5 and 30 min. The rheology tests were carried out at temperature $20 \pm 2{ }^{\circ} \mathrm{C}$. The rotational rheometer Rheotest RN 4.1 with coaxial cylinders was used for rheological tests. The scheme of cylinder measuring system of rheometer is presented in Figure 2a. The assembled measuring cup (1) with coupling is poured by cement paste (3), and fixed in the equipment stand. The rotating cylinder rotor (2) is placed inside the measuring cup (1) with gap between them of $1.5 \mathrm{~mm}$ and rotates during the test. Shear rate ranged from $100.0 \mathrm{~s}^{-1}$ to $0.1 \mathrm{~s}^{-1}$ during testing time. The shear rate mode presented in Figure $2 b$ was used in the current experiments.

Cement pastes after mixing between tests were placed in the plastic containers and carried in the laboratory conditions with temperature $20 \pm 2{ }^{\circ} \mathrm{C}$ and relative humidity not less than $65 \%$. The cement pastes were premixed before rheological test by hands in plastic containerusing metallic scoop for $1 \mathrm{~min}$.

a)

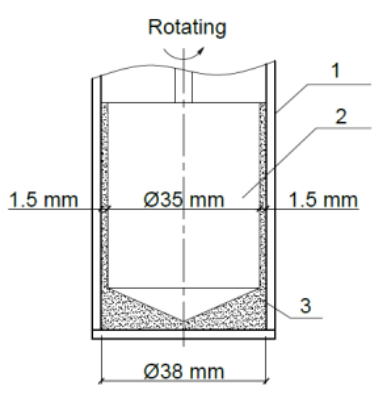

b)

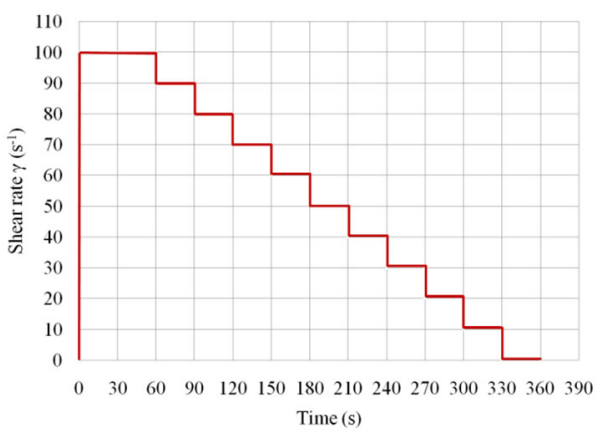

Figure 2. Scheme of cylinder measuring system Rheotest RN 4.1 (a) and shear rate mode of rheological test (b)

The yield stress $\left(\tau_{0}\right)$ and plastic viscosity $(K)$ were determined in the course of approximation of flow curve (dependence between shear stress $(\tau)$ and shear rate $(\gamma)$ in the range from 0.1 to $100 \mathrm{~s}^{-1}$ based on Herschel-Bulkley model. The Herschel-Bulkley model is more widely used model for description of flow behavior of cement systems, which is expressed by the Eq. (2):

$$
\tau=\tau_{0}+K \cdot \gamma^{n},
$$

where: $\tau$ - shear stress, $\mathrm{Pa} ; \tau_{0}$ - yield stress of the cement paste, $\mathrm{Pa} ; K$ - plastic viscosity, Pa.s; $\gamma-$ shear rate, $\mathrm{s}^{-1} ; n-$ shear thinning or shear thickening index if $n<1$ or $n>1$, respectively (Yahia, Mantellato, \& Flatt, 2016).

Amount of bleeding water was determined according to the following method. $50 \mathrm{~g}$ of MWCNT suspension waspour into the 100 mlcontainer, after that $50 \mathrm{~g}$ of cement was added for $1 \mathrm{~min}$. Subsequently, MWCNT suspension and cement were mixed continuously for $4 \mathrm{~min}$ in container and were poured into a graduated cylinder of $30 \mathrm{ml}$. The graduated cylinder with cement paste was placed onto the table and the initial volume of cement paste was written. The graduated cylinder was standing without moving and shaking during the test.

The volume ofsedimentedcement in cement paste after water bleedingwas determined after 2 hours since the reference point and every $30 \mathrm{~min}$ after it. The observation of water bleeding was performed till the moment when the volume of cement paste did not change. Water separation (water bleeding) was calculated according the Eq. (3):

$$
K=\frac{a-b}{a} \cdot 100
$$

where: $K$ - coefficient of water bleeding, $\% ; a$ - initial volume of cement paste, $\mathrm{cm}^{3} ; b$ - volume of sedimentedcement, $\mathrm{cm}^{3}$. 


\section{Results and discussion}

\section{Analysis of MWCNT suspension homogenity}

The results of particle size analysis of MWCNT suspensions subjected to ultrasonicaion and rotor-stator homogenization are presented in Figure 3a.

The standard mixer agitation during $5 \mathrm{~min}$ are not enough to distribute the particles with nanometer sizeand elongated shape. The average particle size diameter of MWCNT suspension reached $869.5 \mathrm{~nm}$ as it is presented in Figure 3a. Ultrasonication for 3 min demonstrated the decrease of average diameter from 869.5 to $300.8 \mathrm{~nm}$, what is the evidence of ultrasonication effectiveness. Ultrasonication for 6 min enables to get the average particle size diameter equal to $245.2 \mathrm{~nm}$. The further increase of ultrasonication time did not revealed the significant changes in particle size distribution. In other words, ultrasonication for 6 min was obtained as optimal homogenization time.

Figure $3 \mathrm{a}$ presents that homogenization by rotor-stator technique for $6 \mathrm{~min}$ allows to obtain the particles with average diameter up to $423.3 \mathrm{~nm}$. The increase of homogenization time to $30 \mathrm{~min}$ did not decrease the average particle size significantly.

a)

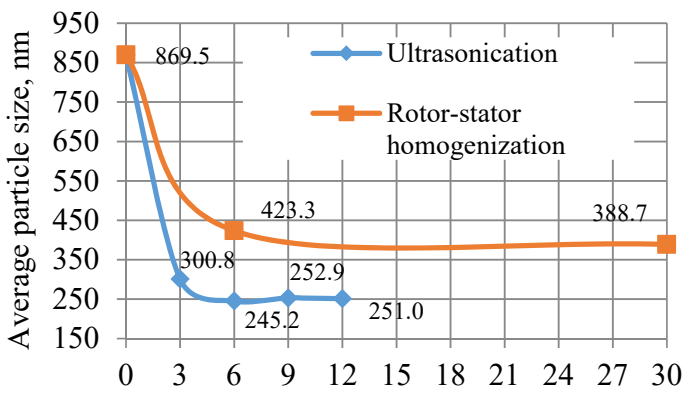

Time of homogenization, min b)

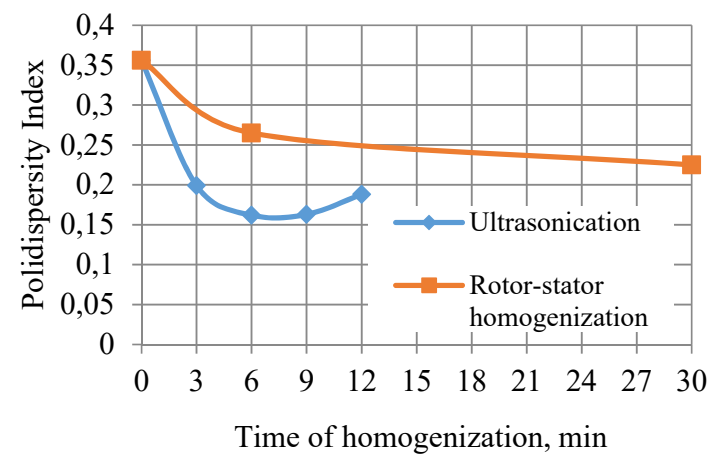

Figure 3. Particle size analysis of MWCNT suspension subjected to ultrasonication and rotor-stator homogenization (a); Polidispersity Index of MWCNT suspensions (b)

As it is seen in Figure 3, the ultrasonication treatment is more effective in comparison with rotor-stator homogenization in chosen time intervals and frequency. The difference in average diameters of particles may be caused by difference in principles of homogenization, which were described above. The changes of polidispersity index (PI), which presents the dimensionless measure of the broadness of the size distribution, are showed in Figure 3b. The value of PI closer to 0 confirms the better homogeneity of nanoparticles in water suspension (Rane \& Choi, 2005). The MWCNT suspension after $6 \mathrm{~min}$ of ultrasonication had the smaller average diameter of particles and PI of 0.15 , which confims the homogeneous distribution.

The results of $\mathrm{pH}$, electrical conductivity, zeta potential and surface tension measurements for MWCNT suspension with different ultrasonication time are available in Table 3.

Table 3. Properties of MWCNT suspensions with different ultrasonication time

\begin{tabular}{|c|c|c|c|c|}
\hline Specimen & $\mathrm{pH}$ & $\begin{array}{c}\text { Electrical conductivity, } \\
\mu \mathrm{S} / \mathrm{cm}\end{array}$ & $\begin{array}{c}\text { Surface tension, } \\
\mathrm{mN} / \mathrm{m}\end{array}$ & $\begin{array}{c}\text { Zeta potential, } \\
\mathrm{mV}\end{array}$ \\
\hline $\mathrm{S} 3$ & 5.07 & 0.0744 & 67 & -52.09 \\
\hline $\mathrm{S} 6$ & 5.05 & 0.0754 & 67 & -51.29 \\
\hline $\mathrm{S} 9$ & 4.84 & 0.0755 & 67 & -54.46 \\
\hline $\mathrm{S} 12$ & 4.87 & 0.0698 & 67 & -53.24 \\
\hline
\end{tabular}

Results presented in Table 3 revealed that $\mathrm{pH}$, electrical conductivity, surface tension and zeta potential of MWCNT suspension subjected to different time of ultrasonication remained on the same level.

To sum up, ultrasonication for 6 min was obtained as optimal homogenization method in the course of the current research. The observation of changes in $\mathrm{pH}$, electrical conductivity, surface tension and zeta potential did not reveal the significant dependency between them and duration of ultrasonication. 


\section{Rheological properties of cement pastes with MWCNT suspension}

Figure 4 and 5 present the flow behavior of cement pastes modified by MWCNT suspension with different duration of ultrasonication. The equations derived based on the Herschel-Bulkley model, which describe the flow behavior of cement pastes, are listed in Table 4 . The thixotropy indexes are listed in Table 4 as well.

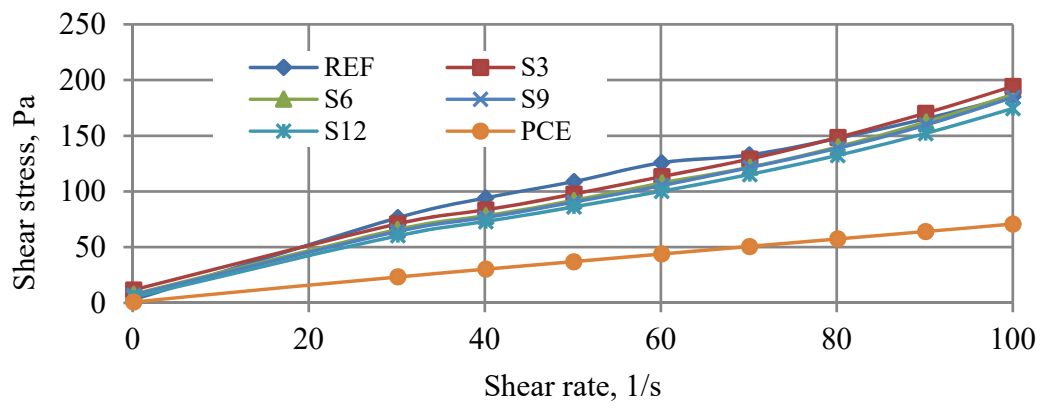

Figure 4. Flow curves of nanomodified cement pastes after 5 min of cement paste mixing

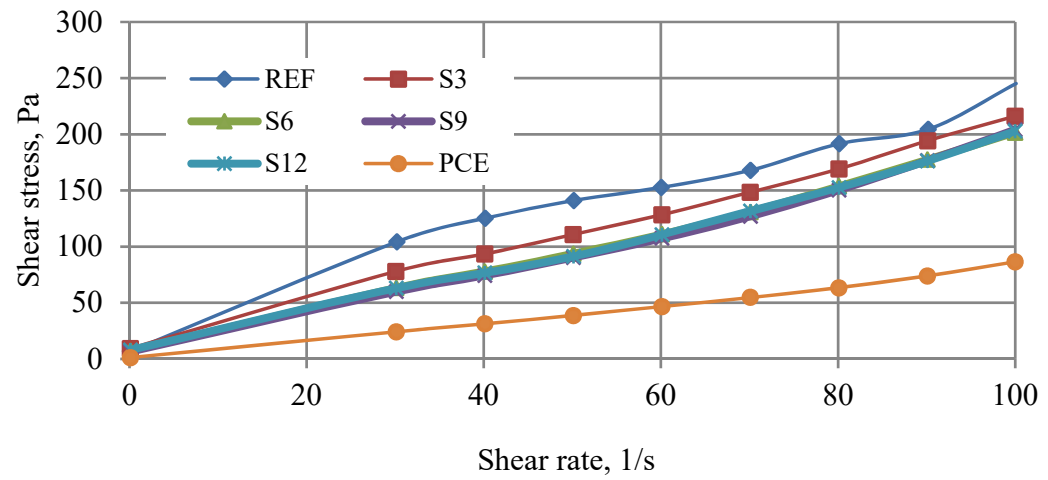

Figure 5. Flow curves of nanomodified cement pastes after 30 min of cement paste mixing

Figure 4 demonstrates that modification of cement pastes by MWCNT suspension leads to the increase of shear stress and consistency factor in comparison with cement pastes modified by PCE in 5 min after mixing of cement pastes. Time of ultrasonication did not influence on rheological behaviour of cement pastes in 5 min after mixing. The cement pastes modified by PCE and MWCNT suspensions with different duration of ultrasonication treatment demonstrated the shear thickening behaviour in comparison with reference sample without admixtures characterized by shear thinning behaviour.

As it seen from Figure 5, the flow behaviour of studied cement pastes did not change significantly in 30 min after their mixing. The duration of ultrasonication treatment did not have the significant effect on the rheological properties of cement pastes in 30 min after their mixing.

Table 4. Low curves equations based on Herschel-Bulkley model and thixotropy indexes of cement pastes

\begin{tabular}{|c|c|c|c|c|}
\hline \multirow{2}{*}{ Specimen } & \multicolumn{2}{|c|}{ Flow curves equations } & \multicolumn{2}{c|}{ Thixotropy Indexes } \\
\cline { 2 - 5 } & After 5 min & After 30 min & After 5 min & After 30 min \\
\hline REF & $\tau=2.96+1.97 \gamma 0.996$ & $\tau=6.9+5.91 \gamma 0.790$ & 0.996 & 0.790 \\
\hline PCE & $\tau=0.85+0.62 \gamma 1.034$ & $\tau=1.24+0.37 \gamma 1.177$ & 1.034 & 1.177 \\
\hline S3 & $\tau=11.75+1.73 \gamma 1.011$ & $\tau=9.3+2.07 \gamma 1.002$ & 1.011 & 1.002 \\
\hline S6 & $\tau=7.8+1.72 \gamma 1.009$ & $\tau=7.2+0.91 \gamma 1.160$ & 1.009 & 1.160 \\
\hline S9 & $\tau=7.2+1.71 \gamma 1.008$ & $\tau=6.5+0.90 \gamma 1.165$ & 1.008 & 1.165 \\
\hline S12 & $\tau=5.3+1.64 \gamma 1.008$ & $\tau=7.98+0.91 \gamma 1.165$ & 1.008 & 1.165 \\
\hline
\end{tabular}

Figure 6a demonstrates the changes in yield stress. The addition of PCE plasticizer led to the decrease of yield stress in comparison with reference sample without plasticizer. Modification of cement systems by MWCNT 
suspensions with different ultrasonication treatment increased the yield stress in comparison with sample modified by PCE plasticizer at the same dosage and reached the values closer to reference cement paste.

The similar rheological behaviour was observed in $30 \mathrm{~min}$ after cement pastes mixing. The addition of MWCNT suspension increased yield stress in comparison with sample modified only by PCE plasticizer. The changes in duration of ultrasonication treatment did not have the significant influence on yield stress of cement pastes in 5 and 30 min after cement pastes mixing.

a)

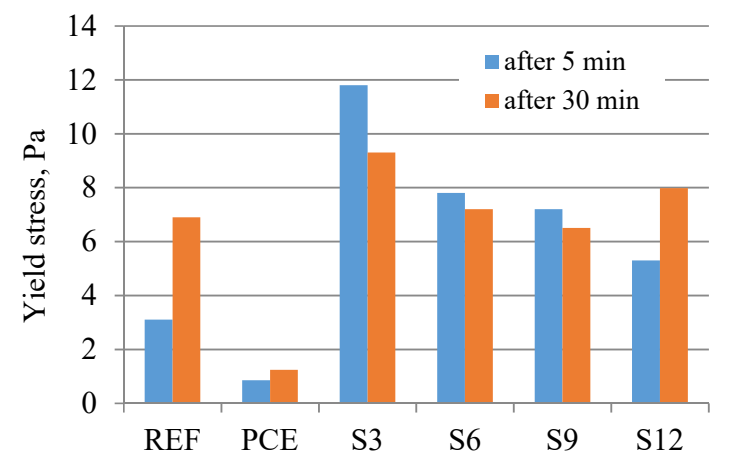

b)

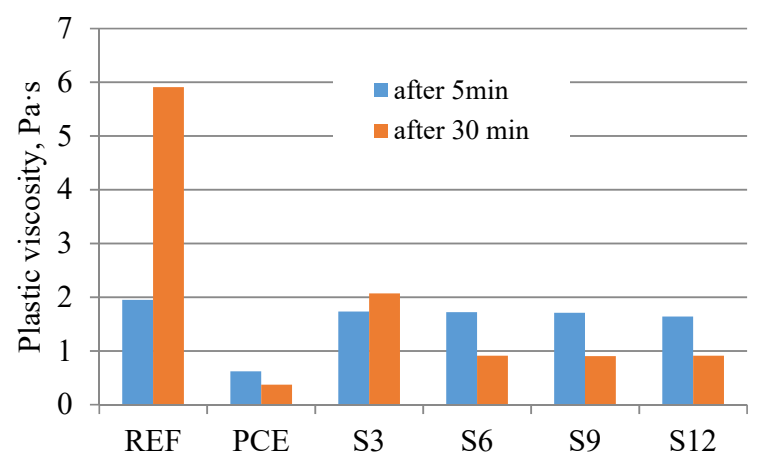

Figure 6. Yield stress (a) and plastic viscosity (b) of nanomodified cement pastes in 5 and 30 min aftermixing

Figure $6 \mathrm{~b}$ presents the changes in plastic viscosity of cement pastes modified by PCE plasticizer and MWCNT suspension. Modification of cement pastes by MWCNT suspension revealed the increase of plastic viscosity. The nanomodified cement pastes in 5 min after mixing had the same plastic viscosity. However, plastic viscosity was different for nanomodfied cement pastes in $30 \mathrm{~min}$ after mixing. The maximum increase of plastic viscosity was observed for cement pastes modified by MWCNT suspension with 3 min ultrasonication treatment. The plastic viscosity increased from 0.37 to $2.07 \mathrm{~Pa} \cdot \mathrm{s}$ in comparison with cement paste modified by PCE.

The increase of yield stress and plastic viscosity of cement pastes modified by MWCNT suspensions with different ultrasonication treatment may be caused by colloidal forces, such as Van-der-Waals, electrostatic and steric forces, which appear between cement particlesin the course of their modification by plasticizer and MWCNT. The time of ultrasonication treatment of MWCNT suspension did not revealed the significant influence on rheological behaviour of cement pastes.

\section{Bleeding of cement pastes modified by MWCNT suspension}

Figure 7 presents the results of bleeding for cement pastes modified by PCE and MWCNT suspensions with different duration of ultrasonication.

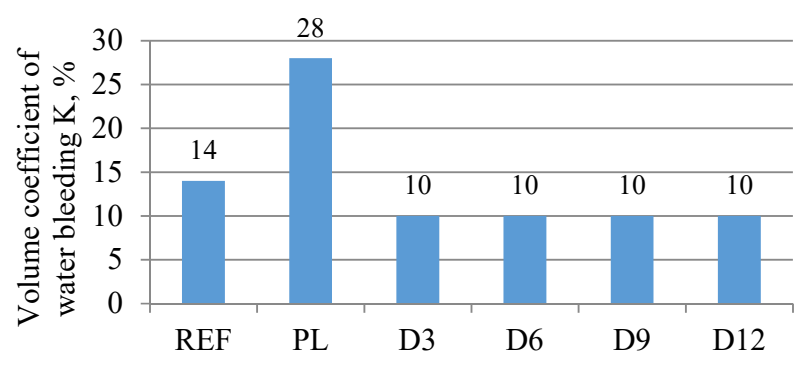

Figure 7. Bleeding of nanomodified cement pastes

Modification by MWCNT suspension leads to decrease of volume coefficient of water bleeding by $4 \%$ in comparison with reference sample without admixtures. Addition of MWCNT enables to decrease the volume coefficient of water bleeding for cement paste with PCE by $18 \%$. The observed changes may be explained by the fine size of MWCNT and their high specific surface.

\section{Conclusions}

The following conclusions are drawn in this study:

1. The particle size was decreased from $869.5 \mathrm{~nm}$ to 388.7 and from $869.5 \mathrm{~nm}$ to $245.2 \mathrm{~nm}$ in the case of rotor-stator homogenization and ultrasonication, respectively. Ultrasonication of MWCNT suspension for 6 min 
was obtained as optimal homogenization method in the course of the current research performed by Bandelin Sonopuls HD 3400 ultrasonic homogenizer $(400 \mathrm{~W}, 20 \mathrm{kHz})$ with probe VS $200 \mathrm{~T}$ (Ø $25 \mathrm{~mm}$, amplitude $82 \mu \mathrm{m})$. The observation of changes in $\mathrm{pH}$, electrical conductivity, surface tension and zeta potential of MWCNT suspensions with different time of ultrasonication treatment did not revealed the significant dependency between them and duration of ultrasonication.

2. The yield stress and plastic viscosity of cement paste modified by MWCNT suspension increased in comparion with cement paste modified by PCE plasticizer in 5 and 30 min after cement pastes mixing. The increase of yield stress and plastic viscosity for cement pastes modified by MWCNT suspensions with different ultrasonication treatment may be caused byVan-der-Waals, electrostatic and steric forces, which appear between cement particlesin the course of modification by plasticizer and MWCNT. Time of ultrasonication of MWCNT suspension more than 6 min did not have the influence on rheological behaviour of cement pastes.

3. Modification by MWCNT suspension leads to decrease of volume coefficient of water bleeding by $4 \%$ in comparison with reference sample without admixtures. Addition of MWCNT enables to decrease the volume coefficient of water bleeding for cement paste with PCE by $18 \%$.

4. Effect of MWCNT suspension on the rheological properties of cement pastes is one of the fundamental step in understanding of their action mechanisms, however the further researches on hydartion kinetics, mechanical properties and durability of nanomodified cement composites are necessary.

\section{References}

Bentz, D. P., Ferraris, C. F., Galler, M. A., Hansen, A. S., \& Guynn, J. M. (2012). Influence of particle size distributions on yield stress and viscosity of cement-flyashpastes. Cement and Concrete Research, 42(2), 404-409. https://doi.org/10.1016/j.cemconres.2011.11.006

Chai, Y. H., Yusup, S., Chok, V. S., Arpin, M. T., \& Irawan, S. (2016). Investigation of thermal conductivity of multi walled carbon nanotube dispersed in hydrogenated oil based drilling fluids. Applied Thermal Engineering, 107, 1019-1025. https://doi.org/10.1016/j.applthermaleng.2016.07.017

Dauksys, M., \& Skripkiunas, G. (2018). Testing of rheological properties of concrete mixtures using a special vibrovisco-meter. Journal of Materials in Civil Engineering, 30(7), 1-8. https://doi.org/10.1061/(ASCE)MT.1943-5533.0002281

García-Taengua, E., Sonebi, M., Hossain, K. M. A., Lachemi, M., \& Khatib, J. (2015). Effects of the addition of nanosilica on the rheology, hydration and development of the compressive strength of cement mortars. Composites Part B: Engineering, 81, 120-129. https://doi.org/10.1016/j.compositesb.2015.07.009

Hisseine, O. A., Basic, N., Omran, A. F., \& Tagnit-Hamou, A. (2018). Feasibility of using cellulose filaments as a viscosity modifyinf agent in self-consolidating concrete. Cement and Concrete Composites, 94, 327-340. https://doi.org/10.1016/j.cemconcomp.2018.09.009

Isik, I. E., \& Ozkul, M. H. (2014). Utilization of polysaccharides as viscosity modifying agent in self-compacting concrete. Construction and Building Materials, 72, 239-247. https://doi.org/10.1016/j.conbuildmat.2014.09.017

Jiao, D., Shi, C., Yuan, Q., An, X., Liu, Y., \& Li, H. (2017). Effect of constituents on rheological properties of fresh concrete - A review. Cement and Concrete Research, 83, 146-159. https://doi.org/10.1016/j.cemconcomp.2017.07.016

Kiu, S. S. K., Yusup, S., Soon, C. V., Arpin, T., \& Samion, S. (2016). Lubricant Enhancement via Hydrodynamic and Acoustic Cavitation. Procedia Engineering, 148, 57-63. https://doi.org/10.1016/j.proeng.2016.06.493

Konsta-Gdoutos, M. S., Metaxa, Z. S., \& Shah, S. P. (2010). Highly dispersed carbon nanotube reinforced cement based materials. Cement and Concrete Research, 40(7), 1052-1059. https://doi.org/10.1016/j.cemconres.2010.02.015

Lavergne, F., Belhadi, R., Carriat, J., \& Ben Fraj, A. (2019). Effect of nano-silica particles on the hydration, the rheology and the strength development of a blended cement paste. Cement and Concrete Composites, 95, 42-55. https://doi.org/10.1016/j.cemconcomp.2018.10.007

Liebscher, M., Lange, A., Schröfl, C., Fuge, R., Mechtcherine, V., Plank, J., \& Leonhardt, A. (2017). Impact of the molecular architecture of polycarboxylate superplasticizers on the dispersion of multi-walled carbon nanotubes in aqueous phase. Journal of Materials Science, 52(4), 2296-2307. https://ink.springer.com/article/10.1007/s10853-016-0522-3

Ma, S., Qian, Y., \& Kawashima, S. (2018a). Experimental and modeling study on the non-linear structural build-up offresh cement pastes incorporating viscosity modifying admixtures. Cement and Concrete Research, 108, 1-9. https://doi.org/10.1016/j.cemconres.2018.02.022

Ma, S., Qian, Y., \& Kawashima, S. (2018b). Performance-based study on the rheological and hardened properties of blended cement mortars incorporating palygorskite clays and carbon nanotubes. Construction and Building Materials, 171, 663-671. https://doi.org/10.1016/j.conbuildmat.2018.03.121

Mendoza Reales, O. A., Arias Jaramillo, Y. P., Ochoa Botero, J. C., Delgado, C. A., Quintero, J. H., \& Toledo Filho, R. D. (2018). Influence of MWCNT/surfactant dispersions on the rheology of Portland cement pastes. Cement and Concrete Research, 107, 101-109. https://doi.org/10.1016/j.cemconres.2018.02.020

Meng, W., \& Khayat, K. H. (2018). Effect of graphite nanoplatelets and carbon nanofibers on rheology, hydration, shrinkage, mechanical properties, and microstructure of UHPC. Cement and Concrete Research, 105, 64-71. https://doi.org/10.1016/j.cemconres.2018.01.001

Nadiv, R., Vasilyev, G., Shtein, M., Peled, A., Zussman, E., \& Regev, O. (2016). The multiple roles of a dispersant in nanocomposite systems. Composites Science and Technology, 133, 192-199. https://doi.org/10.1016/j.compscitech.2016.07.008 
Qian, S., Yao, Y., Wang, Z., Cui, S., Liu, X., Jiang, H., Guo, Z., Lai, G., Xu, Q., \& Guan, J. (2018). Synthesis, characterization and working mechanism of a novel polycarboxylate superplasticizer for concrete possessing reduced viscosity. Construction and Building Materials, 169, 452-461. https://doi.org/10.1016/j.conbuildmat.2018.02.212

Rane, S. S., \& Choi, P. (2005). Polydispersity index: how accurately does it measure the breadth of the molecular weight distribution?. Chemistry of Materials, 17(4), 926. https://doi.org/10.1021/cm048594i

Roussel, N., Lemaître, A., Flatt, R. J., \& Coussot, P. (2010). Steady state flow of cement suspensions: A micromechanical state of the art. Cement and Concrete Research, 40(1), 77-84. https://doi.org/10.1016/j.cemconres.2009.08.026

Secrieru, E., Cotardo, D., Mechtcherine, V., Lohaus, L., Schröfl, C., \& Begemann, C. (2018). Changes in concrete properties during pumping and formation of lubricating material under pressure. Cement and Concrete Research, 108, 129-139. https://doi.org/10.1016/j.cemconres.2018.03.018

Smirnova, O. (2018a). Evaluation of superplasticizer effect in mineral disperse systems based on quarry dust. International Journal in Civil Engineering and Technology, 9(8), 1733-1740.

Smirnova, O. (2018b). Development of classification of rheologically active microfillers for disperse systems with Portland cement and superplasticizer. International Journal of Civil Engineering and Technology, 9(10), 1966-1973.

Štefančič, M., Mladenovič, A., Bellotto, M., Jereb, V., \& Završnik, L. (2017). Particle packing and rheology of cement pastes at different replacementlevels of cement by $\alpha-\mathrm{Al}_{2} \mathrm{O}_{3}$ submicron particles.Construction and Building Materials, 139, $256-266$. https://doi.org/10.1016/j.conbuildmat.2017.02.079

Vance, K., Kumar, A., Sant, G., \& Neithalath, N. (2013). The rheological properties of ternary binders containing Portland cement, limestone, and metakaolin or fly ash. Cement and Concrete Research, 52, 196-207. https://doi.org/10.1016/j.cemconres.2013.07.007

Yahia, A., Mantellato, S., \& Flatt, R. J. (2016). Concrete rheology: Abasis for understanding chemical admixtures. In Science and Technology of Concrete Admixtures (Chapter 7, pp. 97-127). WoodheadPublising. https://doi.org/10.1016/B978-0-08-100693-1.00007-2

Yoshio, S., Tatami, J., Yamakawa, T., Wakihara, T., Komeya, K., Meguro, T., Aramaki, K., \& Yasuda, K. (2011). Dispersion of carbon nanotubes in ethanol by a bead milling process. Carbon, 49(13), 4131-4137. https://doi.org/10.1016/j.carbon.2011.05.033

Zhang, Y., Luo, X., Kong, X., Wang, F., \& Gao, L. (2018). Rheological properties and microstructure of fresh cement pastes with varied dispersion media and superplasticizers. Powder Technology, 330, 219-227. https://doi.org/10.1016/j.powtec.2018.02.014

Zou, B., Chen, S. J., Korayem, A. H., Collins, F., Wang, C. M., \& Duan, W. H. (2015). Effect of ultrasonication energy on engineering properties of carbon nanotube reinforced cementpastes. Carbon, 85, 212-220. https://doi.org/10.1016/j.carbon.2014.12.094 